International Journal of Applied Mathematics

Volume 33 No. $1 \quad 2020,59-73$

ISSN: 1311-1728 (printed version); ISSN: 1314-8060 (on-line version)

doi: http://dx.doi.org/10.12732/ijam.v33i1.6

\title{
DRIVEN HARMONIC OSCILLATOR BY TRAIN OF CHIRPED GAUSSIAN PULSES
}

\author{
S.S. Hassan ${ }^{1,2}$, R.A. Alharbey ${ }^{3}$, \\ T. $\operatorname{Jarad}^{2}$, S. Almaatooq ${ }^{1}$ \\ ${ }^{1}$ University of Bahrain \\ College of Sci., Dept. of Mathematics \\ P.O. Box 32038, Kingdom of BAHRAIN \\ ${ }^{2}$ Dept. of Computing and Mathematics \\ Manchester Metropolitan University \\ Manchester M1 5GD, UK \\ ${ }^{3}$ King Abdulzziz University \\ Fac. of Sci., Mathematics Dept. \\ P.O. Box 42696, Jeddah 21551 \\ Kingdom of SAUDI ARABIA
}

\begin{abstract}
Exact analytical operator solutions of the interacting model of a single quantized (non-dissipative) harmonic oscillator (HO) with a train of $n$-chirped Gaussian pulses are derived in terms of the error function of complex argument. Explicit expressions are then calculated and examined computationally for the average photon number of the $\mathrm{HO}$ and the emitted spectrum. The chirp parameter $(c)$ induces non-sinusoidal oscillations that lead to: (i) 'step-like plateau' in the dynamics of the average photon number with both $n, \tau_{R}$ (repetition time) large, and, (ii) a 'hole burning' profile and asymmetrical ringing in the spectrum, depends on the initial state of the HO.
\end{abstract}

AMS Subject Classification: $81 \mathrm{~V} 80,81 \mathrm{~S} 22$

Key Words: harmonic oscillator, chirped Gaussian pulse, spectrum

Received: August 7, 2019

(C) 2020 Academic Publications

${ }^{\S}$ Correspondence author 


\section{Introduction}

Basic statistical quantum mechanical study (e.g. [1], [2]) of radiation-matter or radiation-radiation coupling has activated the research, both theoretically and experimentally, in topical subjects, such as spectroscopy [3]-[6] quantum computation and communication and optical signal information processing (e.g. [7], [8]). The specific model of a single mode of the quantized radiation field modelled as a quantized harmonic oscillator (HO) coupled with short laser pulses is a one fundamental and interesting example to investigate for at least two reasons:

(i) The single HO models a very large $(\infty)$ - number of Rydberg atoms surrounded by a thermal or squeezed vacuum radiation reservoirs [9]-[11].

(ii) The perturbed HO is a suitable physical model of nano-biosensors in coherent phonon manipulation with mechanical resonators [12],[13].

In addition to to our earlier study of pulsed excitation of the single $\mathrm{HO}$ in the cases of multimode rectangular, $\sin ^{2}$ - and exponential laser pulses [14]-[17], we extend here our study to the case where the exciting pulsed laser field is a train of chirped $n$-Gaussian pulses. We examine particularly the dynamical behaviour of the average photon number of the (non-dissipative) HO, as well the nature of the transient scattered radiation subject to variation of the chirped pulses' parameters.

The paper is presented as follows. In Section 2, we present the system Hamiltonian and the analytical solutions of the corresponding model equations. In Sections 3 and 4, we calculate the expressions with the computational results for the average photon number and the spectrum, respectively. In Section 5, a summary is given.

\section{Hamiltonian and model equations}

The system of a single mode quantized $\mathrm{HO}$ coupled to a classical laser pulse has the following operator Hamiltonian model (in units of $\hbar=1$ ) [15] (we use ^ notation to denote quantum mechanical operators [18]),

$$
\hat{H}=\omega_{o} \hat{a}^{\dagger} \hat{a}+\Omega_{o}\left(f(t) \hat{a}^{\dagger} e^{-i \omega_{L} t}+\text { h.a. }\right) .
$$

The notations are: $\omega_{o}$ is the circular frequency of the HO, $f(t)$ is the complex pulse shape of circular frequency $\omega_{L}$ and $\Omega_{O}$ is the pulse strength parameter 
(Rabi frequency associated with the driving pulse). The operators $\hat{a}, \hat{a}^{\dagger}$, are the annihilation and creation operators, respectively, of the HO obeying the commutator relations [18],

$$
\begin{gathered}
{\left[\hat{a}, \hat{a}^{\dagger}\right]=1} \\
{\left[\hat{a}, \hat{a}^{\dagger} \hat{a}\right]=\hat{a}} \\
{\left[\hat{a}^{\dagger}, \hat{a}^{\dagger} \hat{a}\right]=-\hat{a}^{\dagger},}
\end{gathered}
$$

where the commutator of any two operators, $[\hat{A}, \hat{B}]=\hat{A} \hat{B}-\hat{B} \hat{A}$.

In eq. (1), the first term represents the free Hamiltonian of the HO (apart from the constant term $\frac{1}{2} \hbar \omega_{o}[18]$ ), while the rest of the terms represent the coupling of the $\mathrm{HO}$ with the classical pulse. Note, the non-operator classical term $\Omega_{o}^{2}|f(t)|^{2}$ representing the free energy of the classical pulse has no effect within the quantization approach.

The time-dependent Heisenberg equation of motion for any operator $\hat{O}$ is: $i \hbar \hat{O}=[\hat{O}, \hat{H}]$, where $\hat{H}$ is the system Hamiltonian. Thus the operators $\hat{a}, \hat{a}^{\dagger}$ using the Hamiltonian (1) obey the following operator differential equations (with $\hbar=1$ ),

$$
\begin{aligned}
\hat{a}^{*} & =-i \omega_{o} \hat{a}-i \Omega_{o} f(t) e^{-i \omega_{L} t}, \\
\hat{a}^{\dagger} & =i \omega_{o} \hat{a}^{\dagger}+i \Omega_{o} f^{*}(t) e^{i \omega_{L} t} .
\end{aligned}
$$

The solutions of (3) for initial time $\left(t_{o}\right)$ and for arbitrary pulse shape $f(t)$ have the forms:

$$
\begin{aligned}
\hat{a}(t) & =e^{-i \omega_{o}\left(t-t_{o}\right)} \hat{A}(t), \\
\hat{a}^{\dagger}(t) & =e^{i \omega_{o}\left(t-t_{o}\right)} \hat{A}^{\dagger}(t),
\end{aligned}
$$

where

$$
\begin{aligned}
\hat{A}(t) & =\hat{a}\left(t_{o}\right)-i \Omega_{o} I(t) e^{-i \omega_{o} t_{o}}, \\
\hat{A}^{\dagger}(t) & =\hat{a}^{\dagger}\left(t_{o}\right)+i \Omega_{o} I^{*}(t) e^{i \omega_{o} t_{o}}
\end{aligned}
$$

with

$$
I(t)=\int_{t_{o}}^{t} f\left(t^{\prime}\right) e^{-i \Delta t^{\prime} d t^{\prime}}
$$

and $\Delta=\omega_{L}-\omega_{o}$ is the frequency detuning parameter. For a train of n-Gaussian pulse of equal width $f(t)$ has the complex form [6], [19], 


$$
f_{n}(\tau)=\sum_{k=1}^{n} e^{-(1+i c)\left(\tau-(k-1) \tau_{R}\right)^{2}}
$$

where $\tau=\frac{t}{\tau_{o}}$ is the normalized time, $\tau_{o}$ is the full width of a pulse at $\frac{1}{2}$ maximum, $\tau_{R}=\frac{T_{R}}{\tau_{o}}$ is the normalized repetition time and $c$ is the chirp (rate) parameter.

In eq. (6), the initial time $t_{o}$ depends on the switch-on of the pulse. Gaussian pulses have smooth switch-on, and not sharp switch-on, as in e.g. rectangular pulses, so we take $t_{o} \rightarrow-\infty$. Hence, inserting eq. (7) into (6) we get,

$$
\begin{aligned}
I_{n} & =\tau_{o} \int_{-\infty}^{\tau=\frac{t}{\tau_{o}}} e^{-(1+i c)\left(\tau^{\prime}-(k-1) \tau_{R}\right)^{2}} e^{-i \Delta_{o} \tau^{\prime}} d \tau^{\prime} \\
& =\tau_{o} \sum_{k=1}^{n} e^{-(1+i c)(k-1)^{2} \tau_{R}^{2}} \int_{-\infty}^{\tau} e^{a \tau^{\prime 2}-2 b_{k} \tau^{\prime}} d \tau^{\prime} \\
& =\sqrt{\frac{\pi}{a}} \frac{\tau_{o}}{2} \sum_{k=1}^{n} e^{-(1+i c)(k-1)^{2} \tau_{R}^{2}} e^{\frac{b_{k}^{2}}{a}}\left[1+\operatorname{erf}\left(\sqrt{a} \frac{t}{\tau_{o}}+\frac{b_{k}}{\sqrt{a}}\right)\right],
\end{aligned}
$$

where $a=1+i c=a_{o} e^{i \theta}\left(a_{o}=\sqrt{1+c^{2}}, \theta=\tan ^{-1}(c)\right), \Delta_{o}=\Delta \tau_{o}, b_{k}=$ $\frac{a}{2}\left(\frac{i \Delta_{o}}{a}-2(k-1) \tau_{R}\right)$ and $\operatorname{erf}(z)=\frac{2}{\sqrt{\pi}} \int_{0}^{z} e^{-z^{\prime 2}} d z^{\prime}$ is the error function of complex $\operatorname{argument}(z)$, see [20]. In the special case of $n=1$ pulse eq. (8) reduces to

$$
I_{1}(t)=\sqrt{\frac{\pi}{a}} \frac{\tau_{o}}{2} e^{b_{1}^{2} / a}\left[1+\operatorname{erf}\left(\sqrt{a} \frac{t}{\tau_{o}}+\frac{b_{1}}{\sqrt{a}}\right)\right],
$$

with $b_{1}=i \frac{\Delta_{o}}{2}$.

In the next two sections, we formulate the analytical expressions for the average photon number and the scattered spectrum, and investigate them computationally.

\section{Photon number dynamics}

Using eqs. (4)-(6), (8) the (quantum) average photon number of the $\mathrm{HO}, \bar{n}(t)=$ $\langle\hat{n}(t)\rangle$ (related to its energy) for arbitrary initial state is given by

$$
\bar{n}(t)=\left\langle\hat{a}^{\dagger}(t) \hat{a}(t)\right\rangle
$$




$$
\begin{aligned}
& =\left\langle\hat{A}^{\dagger}(t) \hat{A}(t)\right\rangle \\
& =\bar{n}_{o}+\Omega_{o}^{2}\left|I_{n}(t)\right|^{2}-i \Omega_{o}\left[\bar{a}_{o}^{\dagger} e^{-i \omega_{o} t_{o}} I_{n}(t)-c . c\right],
\end{aligned}
$$

where $\bar{n}_{o}=\left\langle\hat{n}\left(t_{o}\right)\right\rangle$ is the initial average photon number, and $\bar{a}_{o}^{\dagger}=\left\langle\hat{a}^{\dagger}\left(t_{o}\right)\right\rangle$ is the initial average value of the $\mathrm{HO}$ amplitude variable. The presence of the factor $e^{-i \omega_{o} t_{o}}$ in (10) modifies the phase of the initial coherent state $\bar{a}_{o}^{\dagger}=\alpha^{*}$. The second term in (10) represents the intensity of the classical driving pulse. Here, we present the computational plots of the normalized photon number, $\bar{n}_{i}(t)=\frac{\langle\hat{n}(t)\rangle_{i}}{\max \langle\hat{n}(t)\rangle_{i}}, i=o, \alpha$, in the two initial states of the HO, namely, the vacuum and coherent states, respectively, where $\max \langle\hat{n}(t)\rangle_{i}$ is the maximum value of $\langle\hat{n}(t)\rangle_{i}$ over the considered time domain.

(a) Initial vacuum state $\bar{n}_{o}(\tau)$

In this case, $\bar{n}_{o}=\bar{a}_{o}=0$ and the normalized form of (10) in this case has the form $\bar{n}_{o}(\tau)=\left|I_{n}(\tau)\right|^{2}$, which is independent of the pulse strength $\Omega_{o}^{2}$. At exact resonance $\left(\Delta_{o}=0\right)$ in the $n=1$ pulse case Fig.1a, $\bar{n}_{o}(\tau)$ reaches its constant maximum value monotonically for $\tau>0$, while for non-zero chirp $|c| \neq 0$, the reach to the lesser constant value is oscillatory. In the off-resonance case $\left(\Delta_{o} \neq 0\right)$ - Fig.1a, $\bar{n}_{o}(\tau)$ has an asymmetric peak for $c=0$ at $\tau=0$. For $c \neq 0$, the constant value for $\tau>0$ is larger with $c<0$. For $\Delta_{o} \neq 0$, and increasing pulse number $n=4, \tau_{R}=4$, where pulses are not overlapping, $c=0$, the step-like structure at $\Delta_{o}=0$ is replaced by pronounced peaks and dips (Fig.1b).

(b) Initial coherent state $\bar{n}_{\alpha}(\tau)$

For initial coherent parameter $\alpha=|\alpha|=5, \Omega_{o}^{\prime}=10, n_{o}=|\alpha|^{2}, \Delta_{o}=$ $3, c=0$ for $n=1$ pulse case, Fig.2a, $\bar{n}_{\alpha}(\tau)$ has the shape of 'Mexican hat' with asymmetry that turns to oscillations for $\tau \lessgtr 0$ when $c>0$. For $c<0$, the oscillations decay faster for $\tau<0$. For larger $n=4, \tau_{R}=4$, Fig.2b, the behaviour is very similar to Fig.1b. in the initial vacuum state, but with distinguishable curves for $c \neq 0$ prior to the constant values.

\section{Transient spectrum}

The transient spectrum of the scattered radiation due the coupled system of $(\mathrm{HO}+n$-chirped Gaussian pulses) is given by [15] 


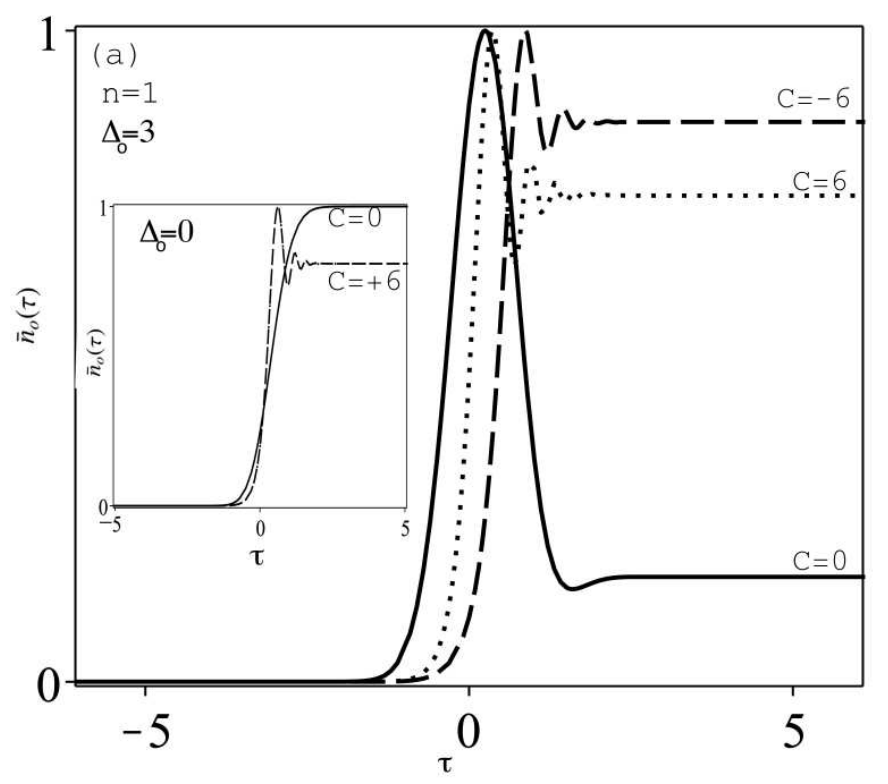

Fig.1a: The normalized average photon number $\bar{n}_{o}(\tau)$, with initial vacuum state of the $\mathrm{HO}$, against the normalized time $\tau=\frac{t}{\tau_{o}}$ for $\Delta_{o}=3, c=0, \pm 6$ and $n=1$ pulse case. Inset figure shows the case for $\Delta_{o}=0$.

$$
S(t, D, \Gamma)=2 \Gamma \int_{t_{o}}^{t} d t_{1} \int_{t_{o}}^{t} d t_{2} e^{(-\Gamma+i D)\left(t-t_{1}\right)} e^{(-\Gamma-i D)\left(t-t_{2}\right)}\left\langle\hat{A}^{\dagger}\left(t_{1}\right) \hat{A}\left(t_{2}\right)\right\rangle
$$

where the average value of the HO auto-correlation function in (11), using (5) is given by

$$
\begin{aligned}
\left\langle\hat{A}^{\dagger}\left(t_{1}\right) \hat{A}\left(t_{2}\right)\right\rangle & =\left\langle\hat{a}^{\dagger}\left(t_{o}\right) \hat{a}\left(t_{o}\right)\right\rangle+\Omega_{o}^{2} I_{n}^{*}\left(t_{1}\right) I_{n}\left(t_{2}\right)-i \Omega_{o}\left\langle\hat{a}^{\dagger}\left(t_{o}\right) I_{n}\left(t_{2}\right)\right\rangle \\
& +i \Omega_{o}\left\langle\hat{a}\left(t_{o}\right) I_{n}^{*}\left(t_{1}\right)\right\rangle
\end{aligned}
$$

with $t_{o} \rightarrow-\infty$ and $\Gamma$ is the radiation detector's width of frequency $\omega$ and $D=\omega-\omega_{0}$ its frequency mismatch. Substituting (12) into (11), we get the following form of the transient spectrum,

$$
S(t, D, \Gamma)=2 \Gamma e^{-2 \Gamma t}\left[n\left(t_{o}\right)\left|J_{1}(t)\right|^{2}+\Omega_{o}^{2}\left|J_{2}(t)\right|^{2}\right.
$$




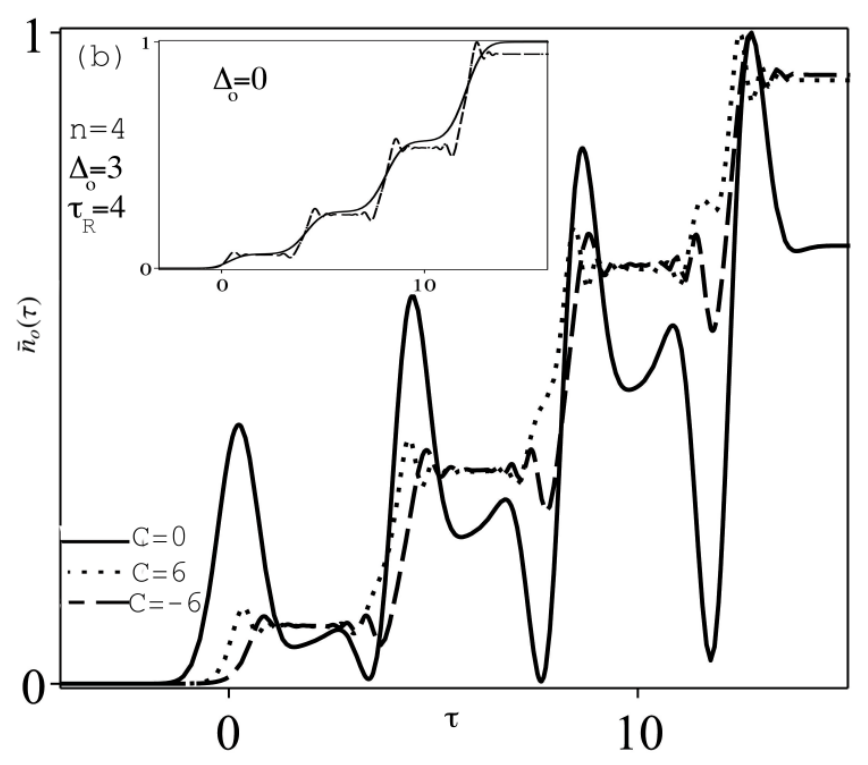

Fig.1b: As Fig.1a but for $n=4$ pulse case and $\tau_{R}=4$. Inset shows the case for $\Delta_{o}=0$.

$$
\left.-2 \Omega_{o} \operatorname{Im}\left(\bar{a}(0) J_{1}^{*}(t) J_{2}(t)\right)\right],
$$

with $\bar{n}_{o}, \bar{a}_{o}$ defined below eq.(9). The quantities $J_{1,2}(t)$ are given by:

$$
\begin{aligned}
& J_{1}(t)=\int_{-\infty}^{t} e^{(\Gamma-i D) t^{\prime}} d t^{\prime}=\frac{e^{(\Gamma-i D) t}}{\Gamma-i D}, \\
& J_{2}(t)=\int_{-\infty}^{t} e^{(\Gamma-i D) t^{\prime}} I_{n}^{*}\left(t^{\prime}\right) d t^{\prime} .
\end{aligned}
$$

This last integral is evaluated by substitution for $I_{n}^{*}(t)$ from (8) and upon integration by parts we finally get

$$
J_{2}(t)=\sqrt{\frac{\pi}{a^{*}}} \frac{\tau_{o}}{2} \sum_{k=1}^{n} e^{\left(b_{k}^{2} / a\right)^{*}} e^{-(1-i c)(k-1)^{2} \tau_{R}^{2}}\left[J_{1}(t)+F_{k}(t)\right],
$$




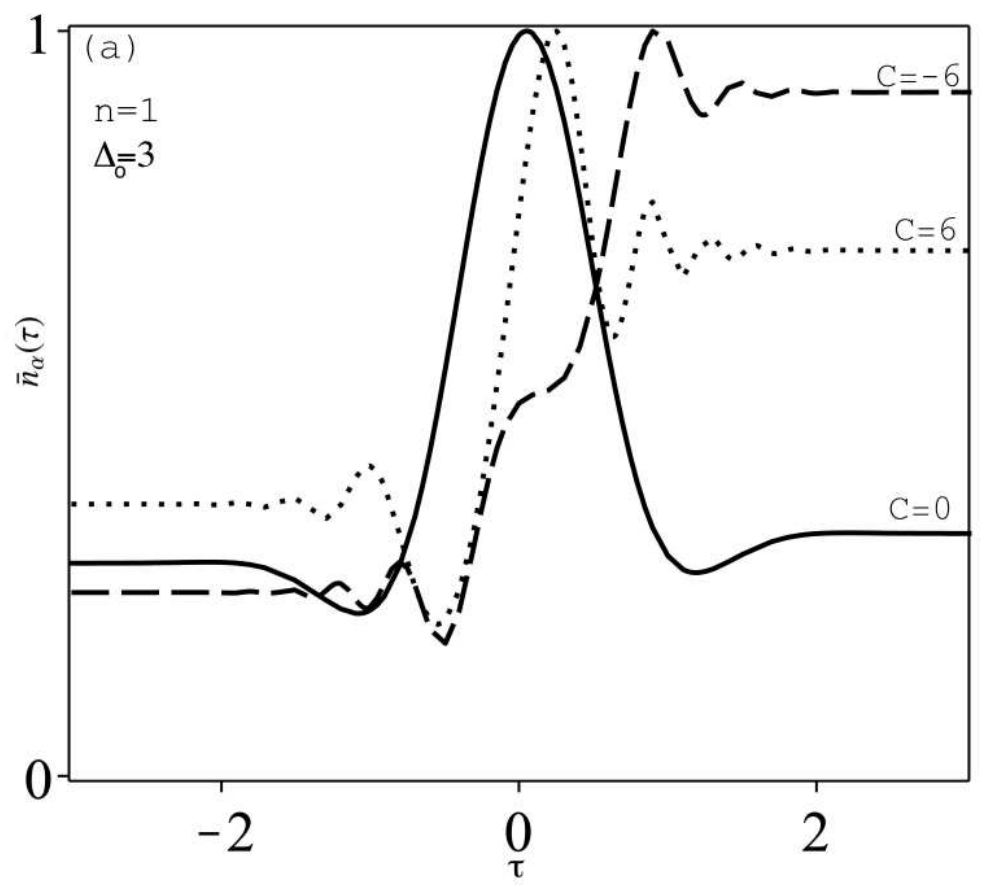

Fig.2a: The normalized average photon number $\bar{n}_{\alpha}(\tau)$, with initial coherent state of the HO, against the normalized time $\tau=\frac{t}{\tau_{o}}$, for $\Delta_{o}=3, c=0, \pm 6, \alpha=5, \theta=0, \Omega_{o}^{\prime}=10$ and $n=1$ pulse case.

where

$$
\begin{aligned}
F_{k}(t) & =\frac{e^{-\beta_{k} \frac{(\Gamma-i D)}{c_{1}}}}{\Gamma-i D}\left\{e^{\frac{(\Gamma-i D)\left(c_{1} t+\beta_{k}\right)}{c_{1}}} \operatorname{erf}\left(c_{1} t+\beta_{k}\right)\right. \\
& \left.-e^{\frac{(\Gamma-i D)^{2}}{4 c_{1}^{2}}}\left[\operatorname{erf}\left(c_{1} t+\beta_{k}-\frac{\Gamma-i D}{2 c_{1}}\right)-f_{1}\right]\right\},
\end{aligned}
$$

with $f_{1}=\lim _{t_{o} \rightarrow-\infty} \operatorname{erf}\left(c_{1} t_{o}+y\right), y=\beta_{k}-\frac{\Gamma-i D}{2 c_{1}}$.

The calculated transient scattered spectrum $S(t, D, \Gamma)$ is given by eq. (13) where the expressions for the quantities $J_{1,2}(t)$ are given by eq. (14)-(16). In what follows, we present the computational plots of the normalized spectrum 


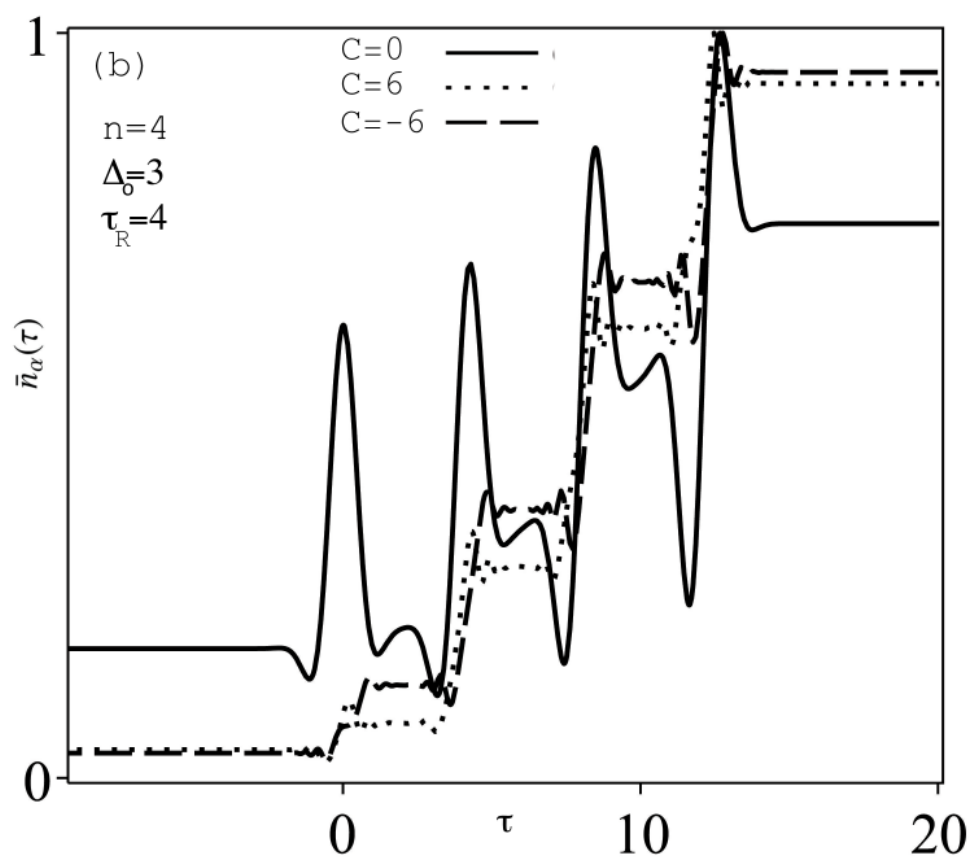

Fig.2b: As for Fig.2a but for $n=4, \tau_{R}=4$

$S_{j}\left(D^{\prime}\right)=\frac{S_{j}(t, D, \Gamma)}{\max S_{j}(t, D, \Gamma)}$ against $D^{\prime}$ for different sets of normalised parameters: $\tau^{\prime}=\Gamma t, \Omega_{o}^{\prime}=\Omega_{o} / \Gamma, D^{\prime}=D / \Gamma, \Delta^{\prime}=\Delta / \Gamma$. The symbol $(j)$ refers to the initial state of the HO. So, $S_{o}\left(D^{\prime}\right), S_{\alpha}\left(D^{\prime}\right)$ refers to the normalised spectra in the initial vacuum and coherent states, respectively.

(i) $n=1$ pulse case

Fig.3a shows that the spectrum $S_{o}\left(D^{\prime}\right)$ for $c=\Delta^{\prime}=0$ is essentially a symmetric Lorenzian. For non-zero values of the chirp and detuning parameters $\left(c, \Delta^{\prime} \neq 0\right)$, the spectrum has an additional prominent peak around $D^{\prime} \approx \Delta^{\prime}$ with oscillatory envelope. The effect of initial coherent state with $\Delta^{\prime} \neq 0$ is reflected in $S_{\alpha}\left(D^{\prime}\right)$ - Fig.3b - as asymmetric hole burning structure at $D^{\prime}=0$, which deepens for $c<0$, with additional small peaks for $\left|D^{\prime}\right| \neq 0$.

(ii) $2 \leq n<20$ pulse case 


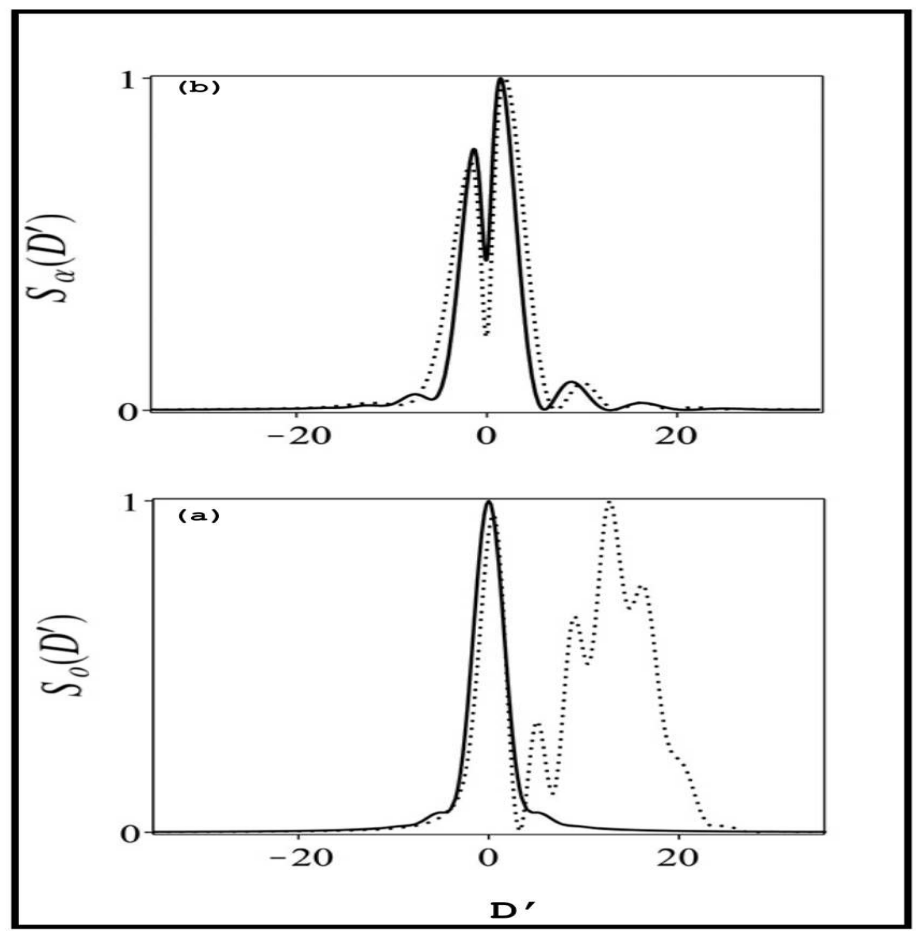

Fig.3: The normalised spectra $S_{o}\left(D^{\prime}\right), S_{\alpha}\left(D^{\prime}\right)$ against the normalised detuning $D^{\prime}$ for $n=1$ pulse case.

(a) The normalised spectrum, $S_{o}\left(D^{\prime}\right)$, in the initial vacuum state for $\tau^{\prime}=0.5 \pi, \tau_{o}^{\prime}=0.3$ and for different $\left(c=0, \Delta^{\prime}=0\right)$ full line, and $\left(c=0.5, \Delta^{\prime}=15\right)$, dotted line.

(b) As (a), but for the initial coherent state, $S_{\alpha}\left(D^{\prime}\right)$, for $\tau^{\prime}=$ $0.3 \pi, \tau_{o}=0.3, \alpha=1, \theta=0.5 \pi, \Delta^{\prime}=8, c= \pm 2$, full and dotted lines, respectively.

Fig.4a shows that for $n=2$ pulse, $\Delta^{\prime}=12, S_{o}\left(D^{\prime}\right)$ has a central peak at $D^{\prime}=0$ with additional smaller peaks for $c>0$ and a prominent peak at $D^{\prime}=\Delta^{\prime}$ for $c<0$. So, the shape of peak structure depends strongly on the sign of $c$. With large $n=10$ pulse - Fig.4b, the dependence of $S_{o}\left(D^{\prime}\right)$ on $c$ is less prominent. The spectrum $S_{\alpha}\left(D^{\prime}\right)$ with initial coherent state for $n=4$ pulse - Fig.4c - has its main structure as: (i) asymmetric narrowing of the central peak, (ii) the extra side peak for $D^{\prime}>0$ is more prominent for $c<0$, and, (iii) occurrence of smaller peaks around the 


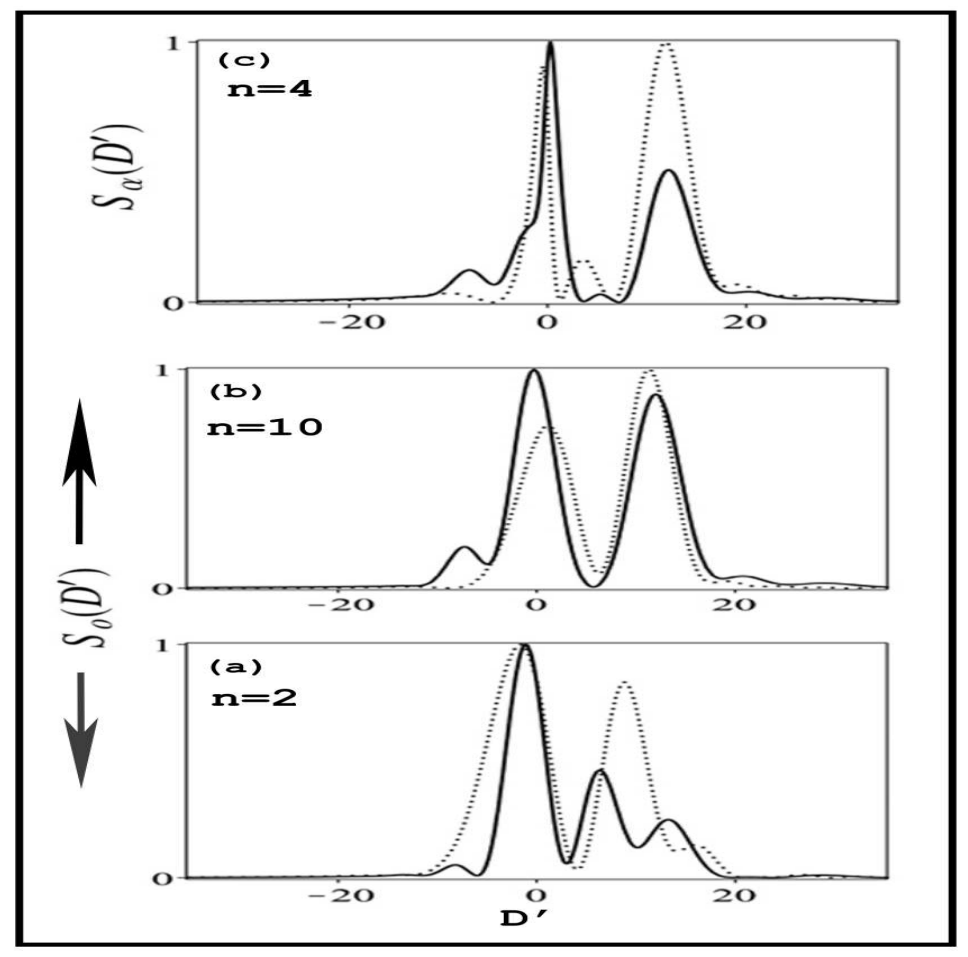

Fig.4: The normalised spectra, $S_{o}\left(D^{\prime}\right), S_{\alpha}\left(D^{\prime}\right)$ against the normalised detuning $D^{\prime}$, for $\tau^{\prime}=0.3 \pi, \tau_{o}^{\prime}=0.3, \tau_{R}=1, \Delta^{\prime}=12, c= \pm 2$, full and dotted lines, respectively.

(a), (b) $S_{o}\left(D^{\prime}\right)$ for $n=2,10$ pulse cases, respectively.

(c) $S_{\alpha}\left(D^{\prime}\right)$, for $n=4$ pulse case and for $n_{o}=1, \Omega_{o}^{\prime}=10$.

central and side peaks at $D^{\prime}=0, \Delta^{\prime}$.

(iii) $n \geq 20$ pulse case

For large number of pulses $n \geq 20$ and large $c=10$ - Fig.5, the spectrum with initial vacuum sate, $S_{o}\left(D^{\prime}\right)$, shows the dependence of the spectrum structure on $\tau_{R}$ (repetition time). For $\tau_{R}=1$, there is asymmetric 3-peak structure at $D^{\prime}=0, \pm \Delta^{\prime}$ with fading ringing for large $\left|D^{\prime}\right|>25$. For $\tau_{R}=$ 0.5 , where the train of pulses merge to the right $(\tau>0)$ as a single pulse of flat peak, the peak for $D^{\prime}<0$ diminishes. For $\tau_{R}>1$, where the pulses are not overlapping, the spectrum $S_{o}\left(D^{\prime}\right)$ is essentially a single symmetric Lorentzian with fading ringing at its tails. The spectrum, $S_{\alpha}\left(D^{\prime}\right)$, with 


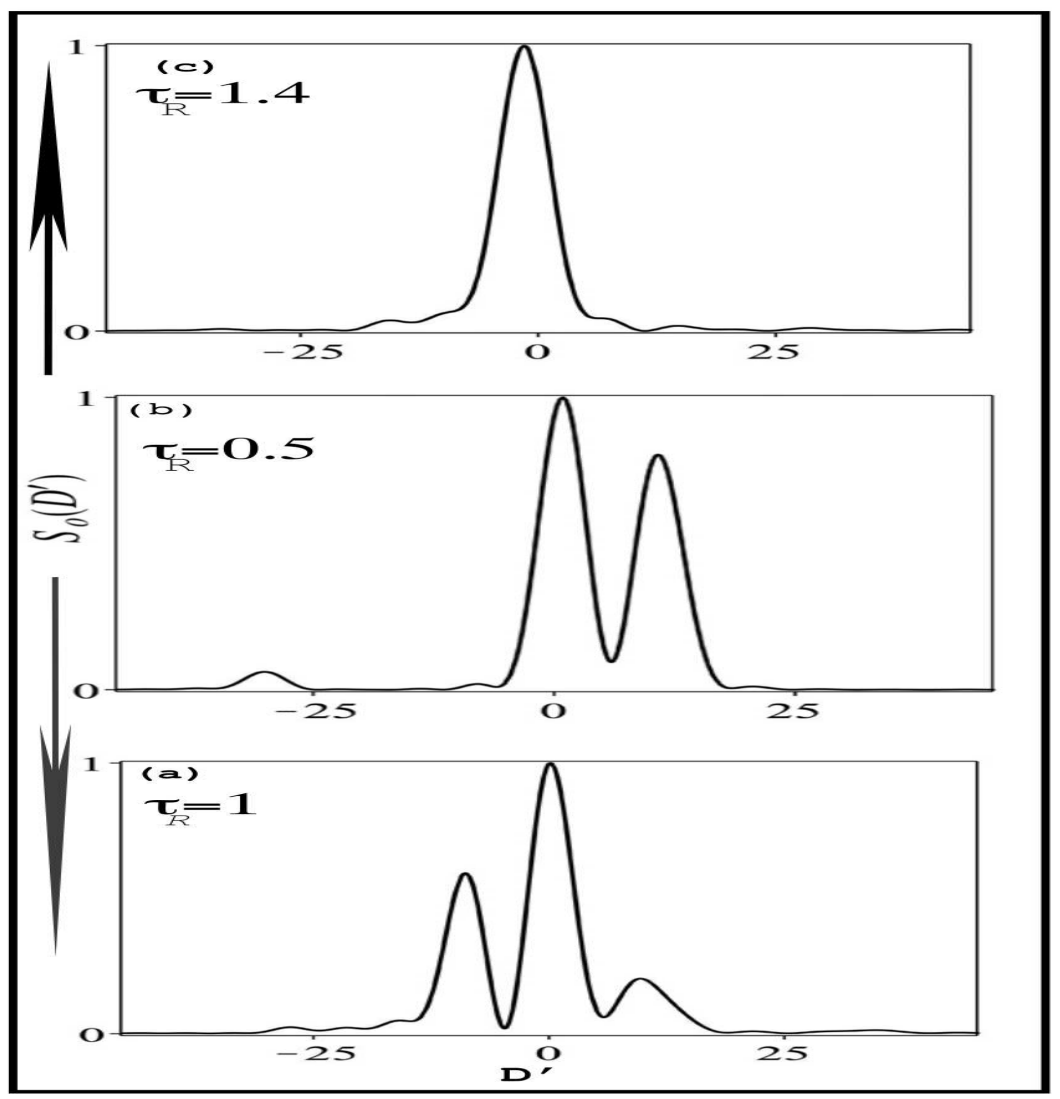

Fig.5: The normalised spectrum, $S_{o}\left(D^{\prime}\right)$, against the normalised detuning $D^{\prime}$, for large $n=20, c=10$ and for $\tau^{\prime}=0.3 \pi, \tau_{o}^{\prime}=$ $0.3, \Delta^{\prime}=12, \tau_{R}=1,0.5,1.4$, (a)-(c), respectively.

initial coherent state show the same feature, broadly speaking, with large $c \geq 10$.

\section{Summary}

We have theoretically investigated the coupling model of a single mode of the (quantized) harmonic oscillator (HO) with train of chirped Gaussian pulses in the absence of damping processes. This concerns the dynamical study of the $\mathrm{HO}$ average photon number (i.e. its average energy) and the transient emitted 
(scattered) spectrum for various system parameters (frequency detuning, Rabi frequency of exciting pulses, chirp parameter, repetition time of pulses) and different initial states of the HO (vacuum and coherent states). Exact analytical solutions of the system variables are obtained in terms of the error function of complex argument. The main results are:

(a) The average photon number dynamics, $\bar{n}(\tau)$

The profile of the chirped n-Gaussian pulses, of chirp and repetition time parameters $c, \tau_{R}$, respectively, together with other system parameters have its reflection on $\bar{n}(\tau)$. Specifically, for $\tau_{R}, n>1$, there is "steplike plateau" structure for $c \neq 0$ (Figs.1,2b) with both initial vacuum and coherent states, respectively.

(b) The transient spectrum, $S(D)$

For a single Gaussian pulse $n=1$, the resonant Lorentzian peak at $c=0$ has additional oscillatory peak with $c \neq 0$ in the initial vacuum state, Fig.3a. For initial coherent state, there is "hole burning" effect - Fig.3b. For both large $n=20, c=10$ - Fig. 5 - the central Lorentzian with initial vacuum state has asymmetrical side peaks for $\tau_{R} \leq 1$, while for $\tau_{R}>1$ it is surrounded by small symmetric ringing. For initial coherent state with both large $n, c$, the spectrum is qualitatively similar to that in Fig.5.

Possible extension of the present model $(\mathrm{HO} \oplus$ train of chirped Gaussian pulses) may include the damping process of the HO in normal [16] and squeezed vacuum reservoirs [21, 22]. Further, inclusion of the fast oscillatory terms, with the following terms replacements:

$$
\begin{aligned}
f(t) e^{-i \omega_{L} t} & \rightarrow 2 f(t) \cos \left(\omega_{L} t\right) \\
f^{*}(t) e^{i \omega_{L} t} & \rightarrow 2 f^{*}(t) \cos \left(\omega_{L} t\right)
\end{aligned}
$$

in eqs. (3a)-(3b), respectively, introduces extra higher frequency terms $e^{ \pm 2 i \omega_{L} t}$ in the analytical solutions [21]-[23], and hence having extra parameter $\frac{2 \omega_{L}}{\gamma} \cong$ $O\left(10^{-7}\right)$, for optical radiation, or, $O(10)$, for microwave radiation. This expects to induce extra oscillations/ringing as in the case of a rectangular pulse [23].

\section{Acknowledgment}

S. S. Hassan acknowledges the hospitality of MMU (July-August, 2019) where the final version of the manuscript was completed. 


\section{References}

[1] J.C. Garrison and R.Y. Chiao, Quantum Optics, Oxford University Press, New York (2008).

[2] G.S. Agarwal, Quantum Optics, Cambridge University Press, Cambridge (2013).

[3] M.O. Scully, and M.S. Zubairy, Quantum Optics, Cambridge University Press, Cambridge, UK (1997).

[4] D. Felinto, C.A.C. Bosco, L.H. Acioli, S.S Vianna, Coherent accumulation in two-level atoms excited by a train of ultrashort pulses, Optics Communications, 215 (2003), 69-73.

[5] Y.B. Band, Light and Matter, J. Wiley, UK (2006).

[6] Y.A. Sharaby A. Joshi and S.S. Hassan, Coherent population transfer in Vtype atomic system, Journal of Nonlinear Optical Physics and Materials, 22 (2013), 1450044-1450059.

[7] W.H. Steeb and Y. Hardy, Problems and Solutions in Quantum Computing and Quantum Information, 2nd Ed., World Sci. Publ., Singapore (2006).

[8] V. Vedral, Introduction to Quantum Information Science, Oxford Univ.Press, Oxford (2008).

[9] S.S. Hassan, G.P. Hildred, R.R. Puri and R.K. Bullough, Incoherently driven Dicke model, S, J. Phys. B, 15 (1982), 2635-2655.

[10] R.K. Bullough, R.R. Puri and S.S. Hassan, Some remarks on the organisation of living matter and its thermal disorganisation, In: Molecular and Biological Physics of Living Systems (Ed. R.K. Mishra), Kluwer Acad. Publ, Netherlands (1990), 1-18.

[11] M.R. Wahiddin, S.S. Hassan, and R.K. Bullough, Cooperative atomic behaviour and oscillator formation in a squeezed vacuum, Journal of Modern Optics, 42 (1995), 171-189.

[12] H. Okamoto, A. Gourgout, C.-Y. Chang, K. Onomitsu, I. Mahboob, E. Yi Chang and H. Yamaguchi, Coherent phonon manipulation in coupled mechanical resonators, Nature Physics, 9 (2013), 480-484. 
[13] P. Snyder, A. Joshi, J.D. Serna, Modeling a nanocantilever-based biosensor using a stochastically perturbed harmonic oscillator, International Journal of Nanoscience, 13 (2014), 145001-145009.

[14] A. Joshi and S.S. Hassan, Resonance fluorescence spectra of a two-level atom and of a harmonic oscillator with multimode rectangular laser pulses, J. Phys. B: At. Mol. Opt. Phys., 35 (2002), 1985-2003.

[15] S.S. Hassan, R.A. Alharbey and H. Al-Zaki, Transient spectrum of $\sin ^{2}$ pulsed driven harmonic oscillator, J. Nonlinear Optical Phys. and Materials, 23 (2014), 1450052-1450066.

[16] S.S. Hassan, R.A. Alharbey and G. Matar, Haar wavelet Spectrum of $\sin ^{2}$ pulsed driven harmonic oscillator, Nonlinear Optics and Quantum Optics, 48 (2016), 29-39.

[17] S.S. Hassan, R.A. Alharbey and T. Jarad, Transient spectrum of pulseddriven harmonic oscillator: damping and pulse shape effects, Nonlinear Optics, Quantum Optics: Concepts in Modern Optics, 48 (2018), 277-288.

[18] P.A.M. Dirac, The Principles of Quantum Mechanics, Oxford Univ. Press, Oxford, 4th Ed. (1981).

[19] H. Tang, T. Nakajima, Effects of the pulse area and pulse number on the population dynamics of atoms interacting with a train of ultrashort pulses, Optics Communications, 281 (2008), 4671-4675.

[20] N.N. Lebedev, Special Functions and Their Application, Dover, New York (1972).

[21] S.S. Hassan, A. Joshi, O.M. Frege and W. Emam, Damping of a harmonic oscillator in a squeezed vacuum without rotating-wave approximation, $A n$ nals of Phys., 322 (2007), 2007-2020.

[22] T. Jarad, M.R. Qader and S.S. Hassan, Harmonic oscillator with Offresonant squeezed reservoir, International Journal of Applied Mathematics, 23 (2010), 1061-1067.

[23] S.S. Hassan and R.A. Alharbey, Fourier and Haar wavelet spectra of a driven harmonic oscillator without rotating wave approximation, Nonlinear Optics and Quantum Optics, 50 (2019), 315-325. 
